\title{
Current concepts on the man- agement of tooth wear: part 2. Active restorative care 1 : the management of localised tooth wear
}
IN BRIEF
- Identifies the need for active restorative intervention for any patient presenting with pathological tooth wear.
- Describes possible approaches to the successful restoration of a dentition presenting with localised pathological tooth wear.
- Discusses how contemporary treatment protocols have been significantly influenced by advances in understanding of adhesive dentistry and occlusion.

\author{
S. B. Mehta, ${ }^{1}$ S. Banerji, ${ }^{2}$ B. J. Millar ${ }^{3}$ and J.-M. Suarez-Feito ${ }^{4}$ \\ VERIFIABLE CPD PAPER
}

This second of the four part series of articles on the current concepts of tooth wear management will focus on the provision of active restorative care, where the implementation of a preventative, passive approach may prove insufficient to meet the patient's expectations, or indeed prove to be sufficiently adequate to address the extent of the underlying pathology to the desired level of clinical satisfaction. The active restorative management of cases presenting with localised tooth wear (of either the anterior, posterior, maxillary or mandibular variety) will be considered in depth in this paper, including a description of the commonly applied techniques and treatment strategies, where possible illustrated by case examples.

\section{THE NEED FOR ACTIVE RESTORATIVE INTERVENTION}

While for many cases of pathological tooth wear, passive management and monitoring may suffice, for a proportion of cases active restorative intervention will become necessary. The cases which require this generally fall into the categories of where:

- There may be aesthetic concerns

\section{CURRENT CONCEPTS ON THE MANAGEMENT OF TOOTH WEAR}

1. Assessment, treatment planning and strategies for the prevention and the passive management of tooth wear

2. Active restorative care 1 : the management of localised tooth wear

3. Active restorative care 2 : the management of generalised tooth wear

4. An overview of the restorative techniques and dental materials commonly applied for the management of tooth wear

\footnotetext{
1,2General Dental Practitioner and Senior Clinical Teacher, Department of Primary Dental Care, King's College London Dental Institute, Bessemer Road, London, SE5 9RW: ${ }^{3 *}$ Professor, Consultant in Restorative Dentistry, Department of Primary Dental Care, King's College London Dental Institute, Bessemer Road, London, SE5 9RW; ${ }^{4}$ Section of Periodontology, Faculty of Dentistry and Medicine, University of Oviedo, Oviedo, Spain ${ }^{*}$ Correspondence to: Professor Brian J. Millar Email:brian.millar@kcl.ac.uk
}

\section{Refereed Paper}

Accepted 14 November 2011

DOI: 10.1038/sj.bdj.2012.48

${ }^{\circ}$ British Dental Journal 2012; 212: 73-82
- Symptoms of pain and discomfort may exist

- There may be functional difficulties

- An unstable occlusion is present ${ }^{1}$

- The rate of tooth surface loss may be of extreme concern to either the dental operator or patient, which furthermore if neglected may culminate in exposure of the dental pulp. ${ }^{2}$

It is prudent, however, to initially implement a preventative programme and a period of monitoring of 6-12 months before embarking upon what usually involves complex, technically demanding restorative dentistry. Such a period of time will not only allow the operator to ensure that the aetiological factor has been eliminated or greatly reduced, but also provide time for the patient to understand the nature of the treatment plan being proposed and importantly, a period of time for the operator to establish a rapport with the patient.

There may however, be special circumstances, such as in cases of aggressive erosion-related wear, where active intervention will need to be implemented more rapidly.

For the purposes of description, active restorative intervention will be subdivided into that for localised wear (maxillary anterior, mandibular anterior, localised posterior) and generalised wear respectively.

\section{LOCALISED MAXILLARY} ANTERIOR TOOTH WEAR

Maxillary anterior teeth are most commonly involved in localised tooth wear, especially where erosion is a major factor.

The decision on how to optimally restore these teeth will depend on the interplay between five factors:

1. The pattern of anterior, maxillary tooth surface loss

2. Inter-occlusal space availability

3. Space requirements of the dental restorations being proposed

4. The quantity and quality of available dental hard tissue and enamel respectively

5. The aesthetic demands of the patient.

The pattern of anterior maxillary tooth wear has been classified by $\mathrm{Chu}$ et $a .^{3}$ into three categories in order to facilitate restorative treatment planning:

1. Tooth wear limited to the palatal surfaces only

2. Tooth wear involving the palatal and incisal edges, with reduced clinical crown height

3. Tooth wear limited to labial surfaces only.

For cases involving visible surfaces such as the latter two categories, restorative techniques will involve the use of tooth coloured aesthetic materials. Metallic restorations, such as metal palatal veneers, 
have little or no place for these cases. In contrast, for cases where the pathology is palatal only, metal palatal veneers may prove to be an excellent restorative option. The merits of each technique and materials used to facilitate the restoration of worn dentitions will be discussed in detail in paper 4 of this series.

\section{Inter-occlusal space availability}

In the majority of patients, tooth wear is accompanied by dento-alveolar compensation. ${ }^{4}$ The latter physiological compensatory mechanism allows occlusal contacts to be maintained, in order to attempt to preserve the efficacy of the masticatory system. However, the possible lack of interocclusal space poses a major dilemma for the restorative dentist. Figure 1 depicts an example of a wear case whereby the loss of tooth tissue has been followed by dentoalveolar compensation and a resultant lack of inter-occlusal clearance.

In some cases, particularly where the rate of tooth wear may be very rapid, or compensatory mechanisms evolve at a relatively slower rate, or in the case of a patient with an anterior open bite, deep overbite or increased overjet adequate space may be available between the upper and lower dentition in centric occlusion (CO) in order to accommodate restorations without the need to reduce healthy tooth tissue aggressively or follow a reorganised approach. Adhesive restorations may simply be bonded into the available space in order to restore form, aesthetics and function, while conventional restorations may require minimal reduction of the affected surfaces.

However, for the majority of cases, adequate inter-occlusal clearance is seldom available. One option would be to follow traditional prosthodontic protocols and to create space to accommodate restorations/restorative materials through the process of tooth reduction and to conform to the existing occlusion. It is well known, however, that the preparation of teeth to receive full coverage indirect restorations may culminate in irreversible loss of pulp vitality. A study by Saunders and Saunders in $1998^{5}$ reported periradicular pathology among 19\% of teeth prepared to receive full coverage indirect restorations. In the case of severely worn teeth, tooth reduction may even in the
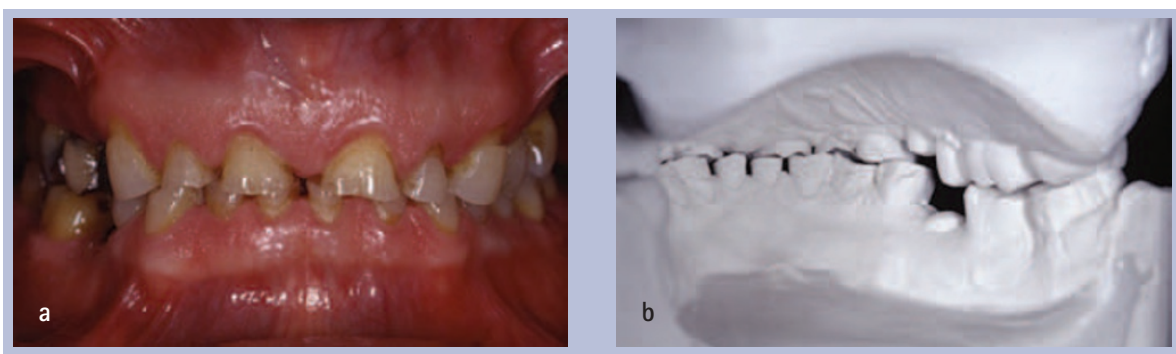

Figs 1a-b An example of a case where tooth wear has been accompanied by dentoalveolar compensation
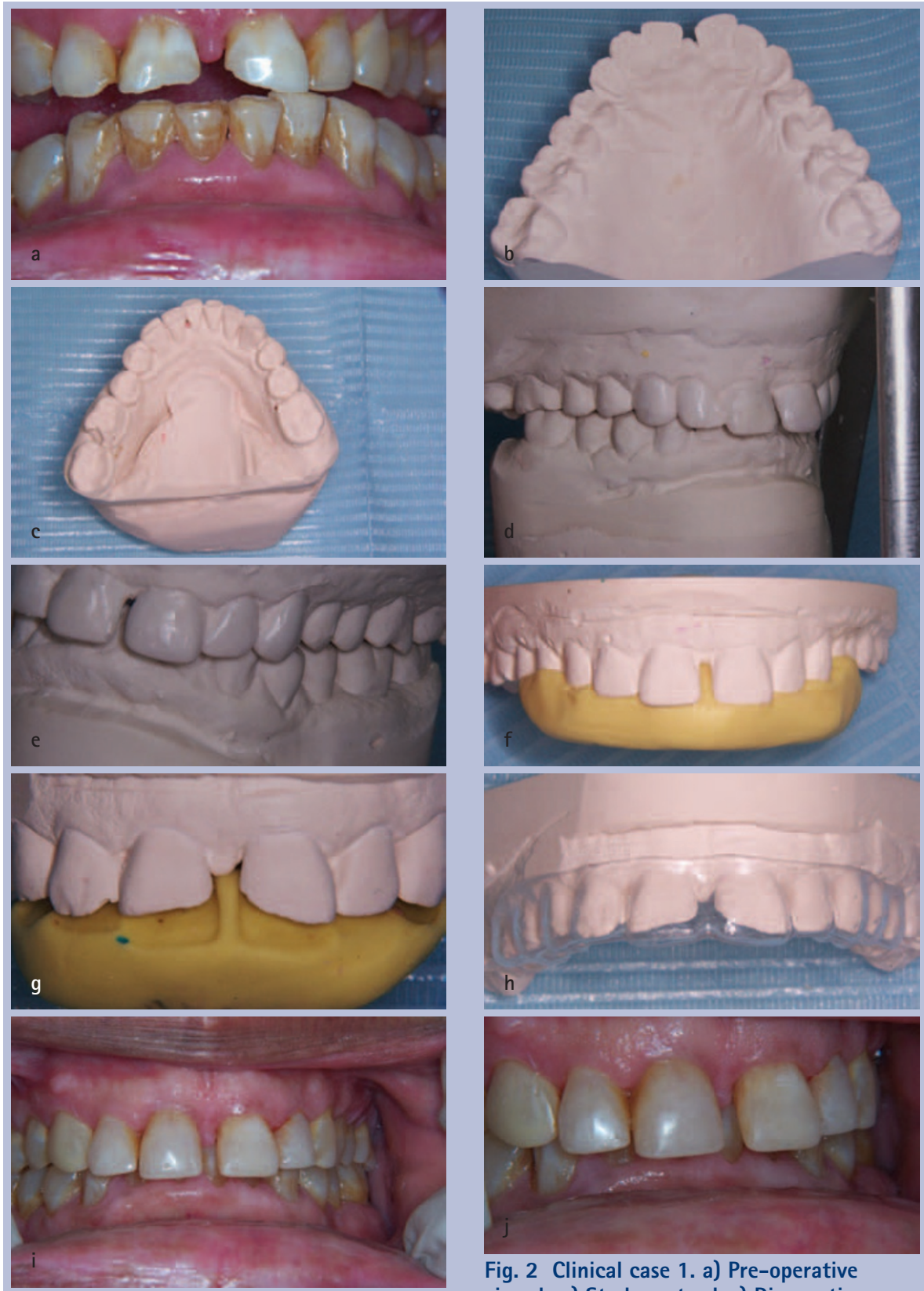

Fig. 2 Clinical case 1. a) Pre-operative view. b-c) Study casts. d-e) Diagnostic wax up to conform to occlusal and aesthetic prescription. $\mathrm{f}-\mathrm{g}$ ) Silicone index (Optosil $P$ plus, Heraeus) produced from post-operative model. h) Vacuum formed thermoplastic template which could also be used as a guide to restore the worn dentition and to display planned changes by using it to carry a provisional crown and bridge material. i-k) Case restored with direct resin composite 
worst scenario lead to iatrogenic pulp tissue exposure.

The aggressive loss of dental hard tissue through tooth preparation, particularly among severely worn teeth, may also lead to a significantly reduced axial height, and thus insufficient retention and resistance form may ensue. It has been suggested by Edelhoff and Sorenssen ${ }^{6}$ that a staggering $62 \%$ to $73 \%$ of tooth structure may be removed during the undertaking of a preparation of a tooth to receive either a full coverage all ceramic crown or porcelain fused to metal crown. While surgical crown lengthening may be an option to consider, this may introduce other undesired effects upon the health of the dental tissues, which will be discussed in more detail in paper 3 of this series.

In some cases, the required inter-occlusal clearance may be present in centric relation (CR). This is often best confirmed by the means of accurate study casts mounted in centric relation on a semi-adjustable (or fully adjustable) articulator, with the aid of diagnostic wax mock-ups. Occasionally, the space created by such a reorganised approach may be sufficient to permit the use of more rigid materials such as metallic alloys which require less bulk thickness to ensure longevity, but not enough for the use of more elastic materials such as resin composite. However, this approach may culminate in several teeth (often unaffected teeth) being in need of restorations in order to maintain occlusal stability, which will further add to the complexity of care, maintenance requirements and procedural cost.

Clinical case 1, as shown in Figure 2, is an example of a case of localised anterior maxillary tooth wear in a 59-year-old male patient who presented with an aesthetic concern. The pattern of wear affected both the palatal surfaces and incisal edges respectively. Following a comprehensive clinical assessment, study casts were taken and mounted on a semi-adjustable articulator. It was decided to stabilise wear using direct composite resin. The rationale for material selection and their respective techniques for successful application will be discussed in detail in paper 4. It is generally accepted that for resin composite restorations to display longevity, in areas of occlusal loading, they should be placed at a minimal thickness of 1.5 to $2.0 \mathrm{~mm}^{7}$
While this level of interocclusal clearance was not available in the intercuspal position, it was ascertained from the mounted casts that adequate space was present in centric relation (without a need to increase the patient's vertical dimension); hence it was decided to restore the worn dentition in a reorganised manner. A diagnostic wax up was undertaken, according to well documented aesthetic principles ${ }^{8}$. The occlusal prescription included contouring the wax build ups to provide even, shared anterior guidance, a canine guided occlusion in lateral excursive and protrusive mandibular movements with posterior tooth separation on dynamic mandibular movements.

A vacuum formed thermoplastic template $(0.5 \mathrm{~mm}$ thickness, Acorn Plastic) was formed from a stone duplicate of the diagnostic wax up. The latter can be used not only to carry a provisional crown and bridge material to the untreated teeth to crudely display planned changes, but also assist in the placement of definitive restoration, to be described in detail in paper 4.

Minimal tooth preparation was undertaken, largely confined to the application of a labial bevel, and the removal of any unsupported enamel with a brown silicone rubber point (Shofu). Placement of resin composite was accomplished with the aid of a silicone matrix formed from the palatal anatomy established from the diagnostic wax up. Resin composite (Filtek Supreme XT, 3M ESPE was applied in layered increments, no greater than $1.5 \mathrm{~mm}$ per increment). Shimstock foil of $8 \mu \mathrm{m}$ thickness (Roeko) was used to assess the patency of the posterior occlusal contacts following the placement of the resin restorations. The patient was advised of the medium term longevity of these restorations, with risks of failure by means of bulk fracture, wear, discoloration and de-bonding. The patient was also warned about possible phonetic changes associated with the bulking out of the cingular areas and lengthening of the anterior maxillary teeth. Alternative methods of resin application will be described in detail in paper 4 . The patient in this case was also provided with a soft maxillary $3 \mathrm{~mm}$ thermoplastic splint for nocturnal use to afford some protection for his newly placed resin restorations.

\section{The Dahl concept}

This concept is frequently referred to in dental literature as a means of gaining space in cases of localised tooth wear, where there is insufficient space available in either CO or CR.

In 1975, Dahl et al. ${ }^{9}$ described the use of a removable anterior bite platform, fabricated from cobalt chromium, retained by clasps in the canine and premolar regions to create inter-occlusal space in a patient with tooth wear localised to the anterior maxillary segment. The appliance was designed to cover the cingulum areas of the affected teeth and increase the occlusal vertical dimension in the region of 2-3 mm.

The placement of this appliance culminated in posterior teeth disclusion; occlusal contacts were only present between the mandibular anterior teeth and the bite platform. The appliance was prescribed for continual wear for several months until the posterior teeth re-established inter-occlusal contact. Removal of the appliance resulted in an inter-occlusal space between the anterior maxillary and mandibular dentitions respectively, which was subsequently utilised to restore the worn surfaces without the need for further tooth reduction.

Dahl and Krungstad ${ }^{10}$ proceeded to repeat the above on a series of patients, and reported that in most cases re-establishment of occlusal contacts occurred within 4-6 months. It has been suggested, however, that it may take up to a period of 18-24 months in some cases (Poyser et al. ${ }^{11}$ ).

The actual Dahl concept refers to the relative axial tooth movement that is observed to occur when a localised appliance or localised restoration(s) are placed in supra-occlusion and the occlusion reestablishes full arch contacts over a period of time. Other phrases used to describe the latter concept include 'minor axial tooth movement', 'fixed orthodontic intrusion', 'localised inter-occlusal space creation' or 'relative axial tooth movement." ${ }^{11}$ The same principle may be extended to the controlled movement of posterior teeth to create space.

The Dahl concept is thought to occur through a process of controlled intrusion and extrusion of dento-alveolar segments. It was reported by Dahl and Krungstad ${ }^{10}$ that the inter-occlusal space created occurs 
through a process of combined intrusion (40\%) and extrusion (60\%). It has also been suggested by Hemmings et al. ${ }^{12}$ that an element of mandibular repositioning involving the condyles may also be occurring concomitantly.

According to a review by Poyser et al., ${ }^{11}$ when considering studies which have assessed the efficacy of the Dahl concept, a success rate of between $94-100 \%$ has been reported. Furthermore, the level of space creation was consistently found to be irrespective of age and sex.

Hemmings et al. ${ }^{12}$ have reported failures to also occur in patients with gross class III malocclusions and in cases with mandibular facial asymmetry that had a lack of stable occlusal contacts in either CO or CR. The lack of eruptive potential is also a factor that should be considered. Patients who may present with bony ankylosis, dental implants, conventional fixed bridgework and those with anterior open bites, will all have limited eruptive potential.

The application of the Dahl concept should also be undertaken with great caution among patients who may have active/a past history of periodontal disease, temporomandibular joint pain dysfunction syndrome, where endodontically teeth may be involved, in cases post-orthodontic treatment (as stability may become compromised) and among patients who may be taking oral or IV bisphosphonate drugs.

Clinical case 2, as shown in Figure 3, is an example of another case of anterior maxillary wear predominantly affecting the palatal surfaces. Space was created with the use of a 'modified' removable cast cobalt-chromium Dahl appliance. The latter appliance was worn continually for a period of two months (other than when eating), and the resultant space used to restore the worn surfaces by the application of adhesive gold palatal veneers (fabricated from Type III cast gold alloy), without the need for any significant tooth preparation, cemented with an adhesive luting agent (Panavia EX, Kuraray, Japan). The occlusal prescription included the provision of a canine guided occlusion, with shared, even anterior guidance on protrusive mandibular movement. The restorations have been successfully in place since 1997. Note the establishment of the posterior occlusal contacts, post-restoratively.
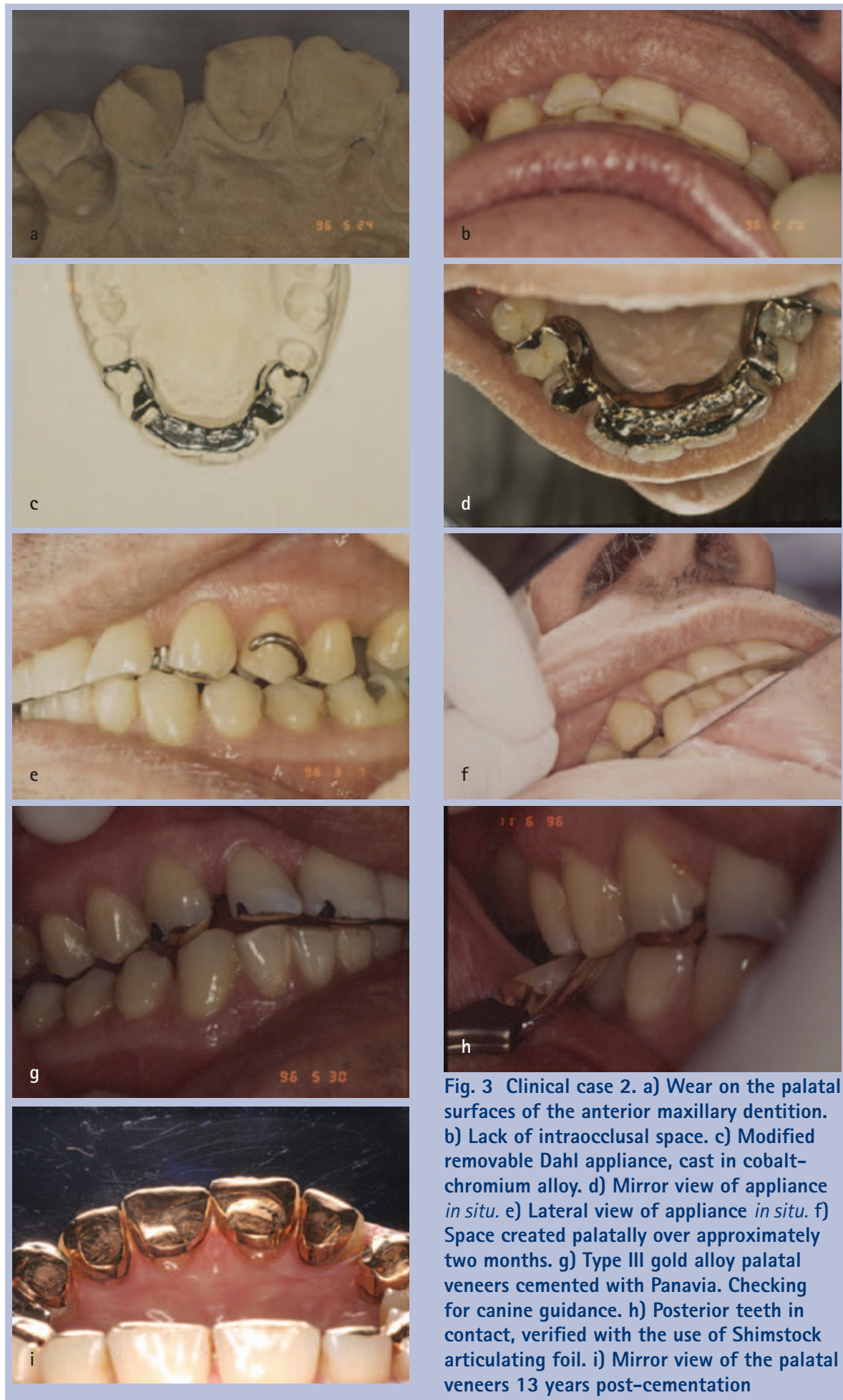

Fig. 3 Clinical case 2. a) Wear on the palata surfaces of the anterior maxillary dentition. b) Lack of intraocclusal space. c) Modified removable Dahl appliance, cast in cobaltchromium alloy. d) Mirror view of appliance in situ. e) Lateral view of appliance in situ. f) Space created palatally over approximately two months. g) Type III gold alloy palatal veneers cemented with Panavia. Checking for canine guidance. $h$ ) Posterior teeth in contact, verified with the use of Shimstock articulating foil. i) Mirror view of the palatal veneers 13 years post-cementation

While there is little evidence in literature to suggest that the process of controlled intrusion and extrusion is associated with possible adverse effects such as pulpal symptoms, periodontal problems, TMJ dysfunction symptoms and apical root resorption, ${ }^{11}$ compliance with removable appliances has been identified as a concern. ${ }^{10}$

To overcome the issues related to patient compliance and aesthetic concerns of the removable prosthesis, Ibbetson and Setchell ${ }^{13}$ have described an alternative approach involving the provision of a fixed metal prosthesis which was cemented in supra-occlusion with the same occlusal prescription as with the removable appliance, with a glass ionomer lute or dual affınity cement. With the subsequent establishment of inter-occlusal clearance, the objective of the treatment plan was to replace the casting with conventional indirect castings. 

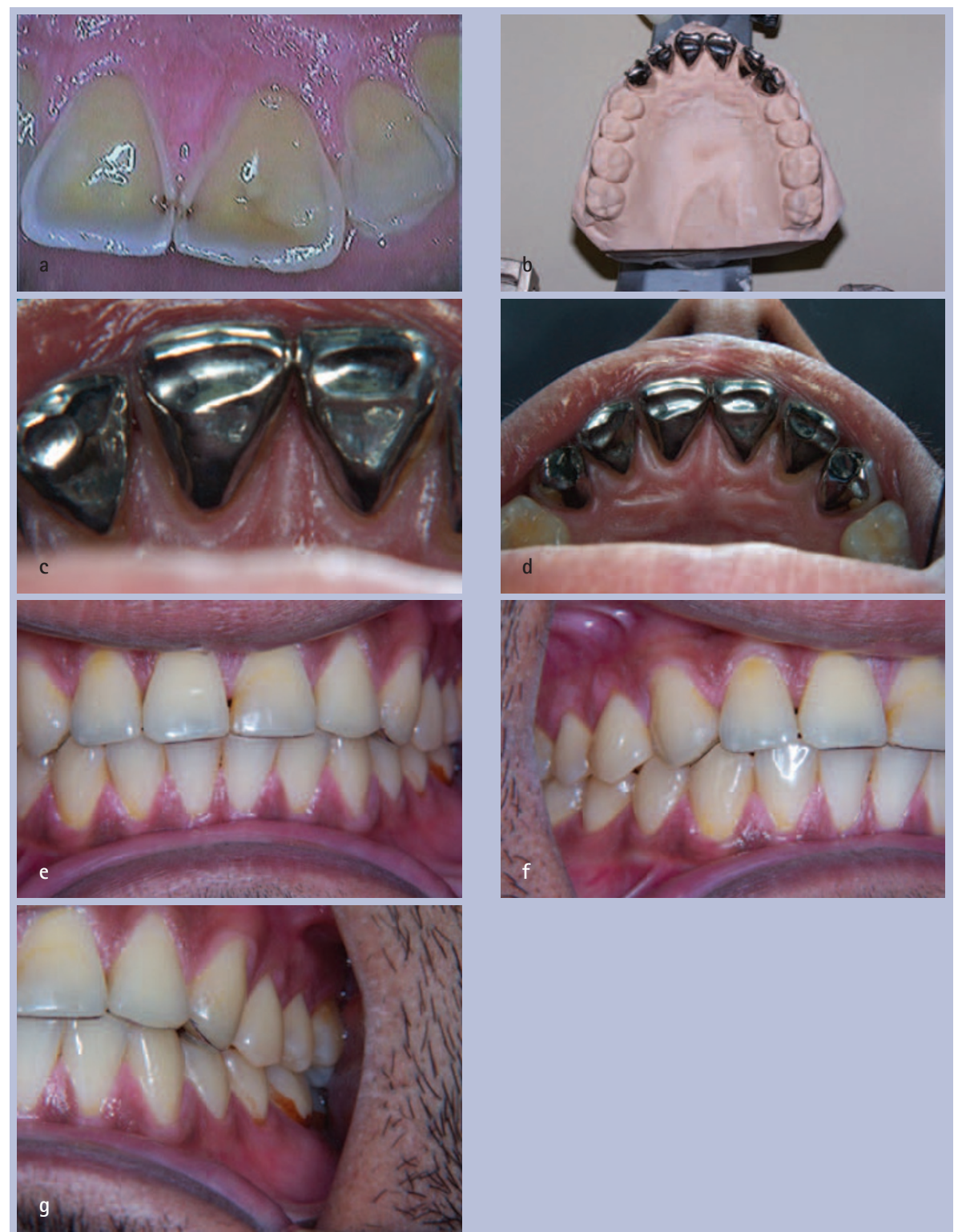

Fig. 4 Clinical case 3. Example of a 'fixed metallic' Dahl appliance. a) Clinical view of Case 3. b) Metal palatal veneers. Note the inclusion of location 'lugs' which can be readily removed post-cementation with the aid of a diamond bur. The resultant rough edge can be smoothed with the use of abrasive discs. c-d) Cemented Ni-Cr veneers in situ. Resin composite has been added to the incisal edge to restore lost tooth tissue and also to help mask the dulling effect at the incisal edge. e-g) Facial view, four years post-operatively. Note the 'dulling' of the anterior maxillary dentition and the visible appearance of metal at the mesial incisal corners of the canine teeth. Composite resin has been added to the incisal edge of UL1, to restore the worn incisal edge and mask the greying effect at this point. Posterior tooth contacts were re-established after a period of three months

Clinical case 3, as depicted in Figure 4, is an example of an anterior maxillary erosive wear case, involving the use of metal (Nickel Chromium) palatal veneers (Dentetch), placed in supra-occlusion utilising the Dahl concept to gain space (hence the concept of a fixed Dahl appliance), in a 24-year-old male patient. Inadequate intra-occlusal clearance was present either in centric occlusion or centric relation; preparation of the affected teeth to accommodate any form of palatal restoration would have undoubtedly culminated in pulp tissue exposure; hence a Dahl approach was taken. Wear was caused by a previous gastric reflux condition. Consent was gained; the patient advised of a risk of intolerance, 'greying' of the restored teeth, initial speech and masticatory difficulties. Veneers were fabricated with a thickness of 0.5-0.7 mm, cemented in supra-occlusion using Panavia 2.0F (Kuraray, Japan). The occlusal prescription included an even/shared anterior guidance and a canine guided occlusion in lateral excursive movements. Restorations were contoured to include the presence of prominent cingular areas to provide a flat occlusal stop for the antagonistic teeth, to help reduce future wear of the lower anterior teeth and to permit the transmission of occlusal loads along the long axes of the involved teeth, with the aim of reducing the likelihood of unwanted labial tooth movement. Posterior tooth occlusal contacts were re-established after a period of three months post-cementation. Due to mild incisal edge wear, and dulling of the upper left central incisor tooth, direct resin composite was applied (Gradia Direct, GC) to restore the latter concerns. This case was restored in 2006.

The removal of the metallic backings may, however, occur at a risk of further compromising an already brittle, worn tooth. Furthermore, the preparation of such teeth to receive conventional restorations may have a negative impact on the pulpal status and the quantity of remaining dental hard tissue. In more recent times more aesthetically pleasing materials have been available, such as composite resin or ceramic. While the initial restorations were designed with metal occlusal platforms in the cingulum area, the use of such platforms is no longer deemed absolutely necessary.

As material technology has continued to evolve, it has now become acceptable to retain the composite resin restorations on anterior teeth, which were in previous times applied on a provisional basis. Such composite restorations may be considered to be 'medium term restorations' (particularly where the wear pattern is largely from erosive causes). It is important that the patient understands that such restorations are vulnerable to fracture, de-bonding, wear and discoloration and may require eventual replacement with indirect restorations.

Figure 5, clinical case 4, provides an example of a wear patient treated utilising the concepts established by the Dahl phenomenon. As there was insufficient interocclusal clearance to accommodate a 1.5 to $2.0 \mathrm{~mm}$ layer thickness of resin composite in either the inter-cuspal position or when the patient was manipulated centric relation, the approach was taken to add material to the affected surfaces in supra-occlusion. A silicone matrix 


\section{PRACTICE}
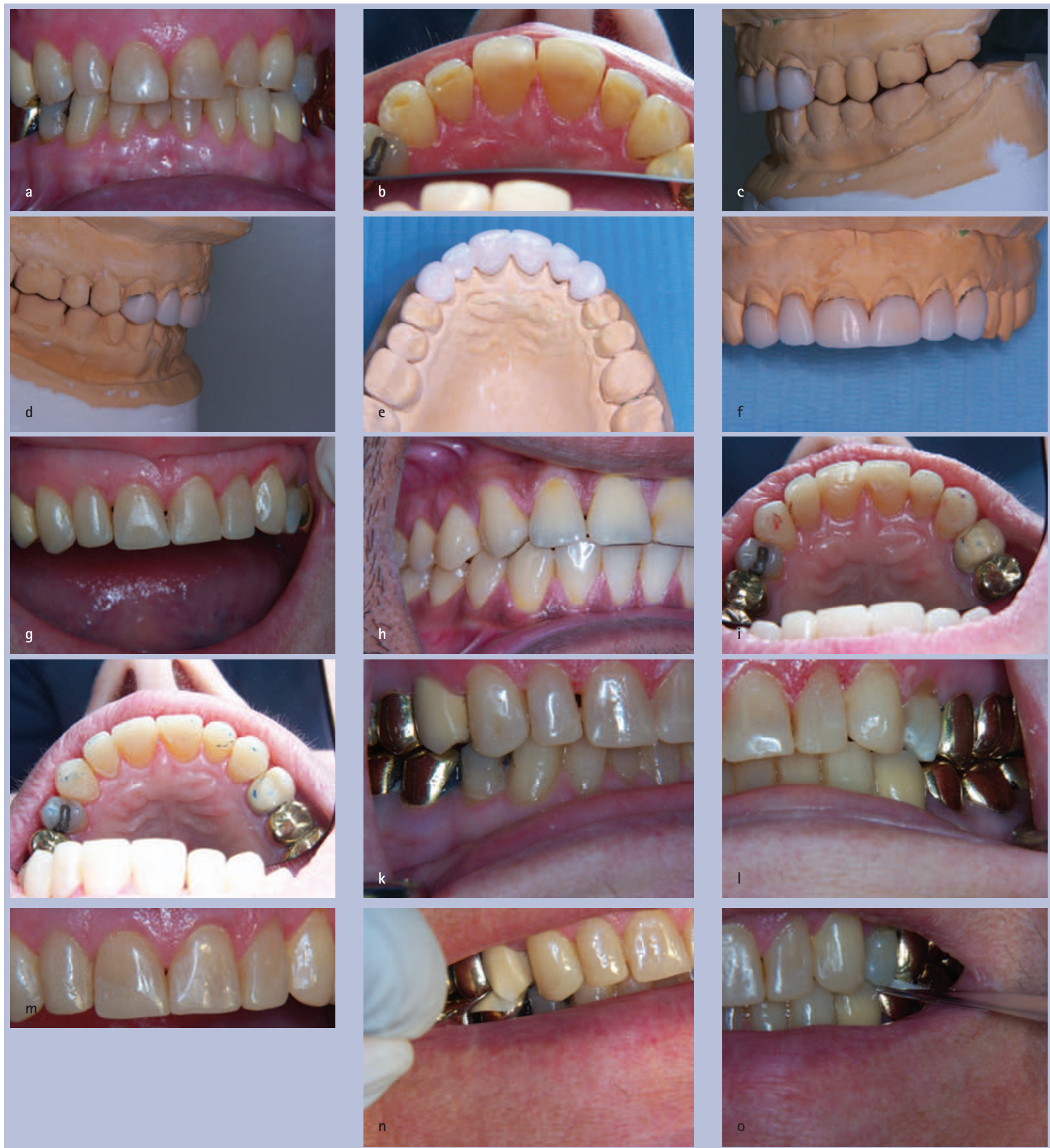

Fig. 5 Clinical case 4. a-b) Pre-operative view. Mild pathological wear affecting the upper anterior dentition due to a combination of bruxism, xerostomia and gastric reflux. c-f) Diagnostic wax up; note disclusion of posterior units. g) Immediate post-operative view following the placement of resin composite (before final polishing phase). $h$ ) The placement of resin composite has been aided with the use of a silicone key formed from the palatal surfaces of the diagnostic wax up. i-j) Post-operative view, to show occlusal form established; even centric stops, canine guided occlusion. $k-1)$ Note separation of posterior teeth immediately following the placement of resin composite. $m-0$ ) Three months post-operative view; note the re-establishment of occlusal stops; after a period of 12 weeks following resin placement

fabricated from a wax up formed from a detailed aesthetic and occlusal prescription was used to aid the application of resin composite. As shown, posterior occlusal stops were re-established after a period of three months post-operatively.
In view of the short to medium term longevity of resin composite restorations, coupled with the aesthetic limitations imposed by such materials, there may be a need to replace such restorations with alternative restorations which may overcome some of the aforementioned limitations. Traditionally, full coverage porcelain fused to metal crowns were applied, however, with advances in dental ceramic technology and improvements in the field of adhesive dentistry respectively, 

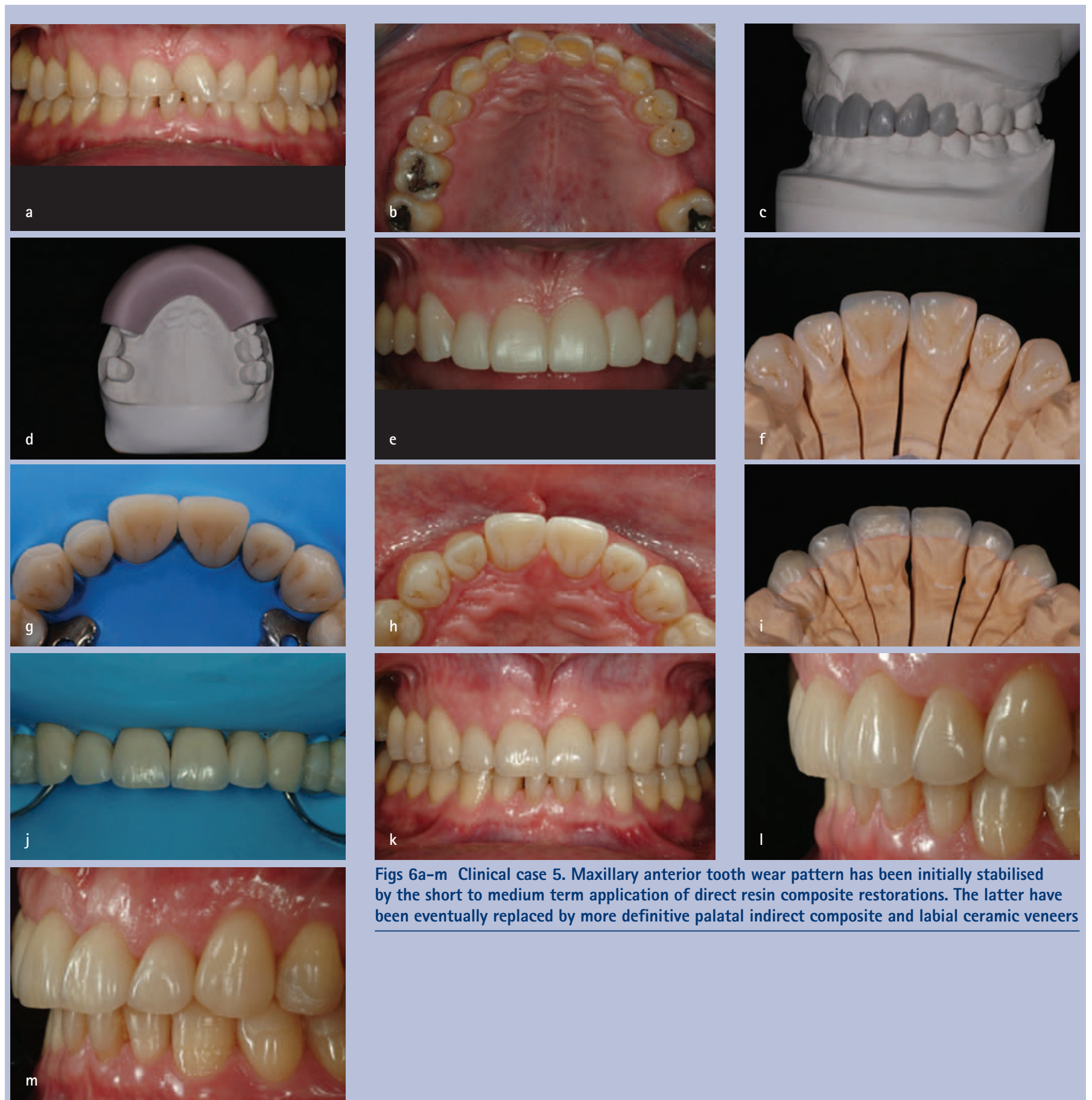

Figs 6a-m Clinical case 5. Maxillary anterior tooth wear pattern has been initially stabilised by the short to medium term application of direct resin composite restorations. The latter have been eventually replaced by more definitive palatal indirect composite and labial ceramic veneers

it is now possible to apply less invasive, more durable, aesthetically superior all ceramic restorations to dentitions where the process of tooth wear has been stabilised by the former application of resin composite, without the need for aggressive tooth reduction.

Figure 6, clinical case 5, is an example of a localised wear case, where resin composite restorations have been placed in supraocclusion to stabilise a wearing dentition. Having attained a stable occlusal scheme and ensured elimination of the aetiological factor(s) causing the observed tooth wear pattern, the direct resin restorations have been replaced by labial feldspathic ceramic veneers and palatally placed indirect nanohybrid resin composite veneers, (placed in a sequential manner). The merits of the latter materials will be discussed in more detail in paper 4 .

Hemmings et $a l .{ }^{12}$ have reported a success rate of $89.4 \%$ for 104 direct hybrid resin composite restorations used to treat localised anterior tooth wear which were placed at an increased vertical dimension. Restorations were followed up for a mean period of 30 months. Patient satisfaction was reported to be good. Occlusal contacts were re-established within the period of four to five months. Splaying of anterior teeth was not reported to be a problematic feature. The authors accounted for the latter observation by the fact that teeth prefer to move through the cancellous trough of bone in a similar way to orthodontic tooth movement, rather than simply in a direct response to the applied occlusal force. ${ }^{14}$ The absence of periodontal disease among the treated teeth is critical in the prevention of splaying. In some cases the authors reported that tooth movement occurred at 
a relatively rapid rate (within one to two months of the placement of the restorations); it was suggested that some element of mandibular repositioning may be responsible. The primary cause of failure was bulk fracture, which (discussed further in paper 4) is an inherent problem associated with the use of resin composite; however, such failures are generally non-catastrophic and readily amenable to repair. The study also showed hybrid composites to perform better than microfilled resins, perhaps related to the reduced rigidity displayed by the latter, which may culminate in increased flexion and subsequent de-bonding upon repeated loading.

Despite advances in resin technology, unfavourable differential wear of direct resin composite against antagonistic teeth and antagonistic restorative materials remains a concern (particularly among wear patients), and for this reason Hemmings et al. ${ }^{12}$ suggest that a reasonable service period of such restorations to be in the range of three to five years. Similar medium term prognostic outcomes have been reported by several other studies, which will be considered in further detail in the subsequent paper. ${ }^{15,16}$

It has been suggested that where a patient is to be treated by the means of a fixed Dahl appliance/restoration, they must be advised of the risks of initial postoperative discomfort, problems of food collection on posterior occlusal surfaces and possible difficulties associated with the eating of certain types of food such as lettuce and ham. ${ }^{12}$ Post-cementation, the occlusal scheme should be reviewed periodically at one, two, six, nine and 12 months respectively post-operatively. ${ }^{12}$

The space requirements of the restorations will be determined by the minimal thickness at which a certain dental material can be applied to the affected surface, in order for it to provide resilient, predictable function. The techniques and materials used to restore worn dentitions are discussed in more detail using case examples as shown below.

In summary space requirements will range from $0.5 \mathrm{~mm}$ for minimally invasive metal palatal veneers, up to $2.0 \mathrm{~mm}$ for ceramic based materials. The provision of this space may necessitate aggressive tooth reduction of an already compromised tooth by way of the quantity of dental hard tissue, but may also compromise the health of the dental pulp. While space may be created by axial tooth movement as described above, there may be some cases where there is a lack of eruptive potential or other contra-indications, as listed above. In such circumstances, a compromised choice may have to be made.

The quantity and quality of remaining dental hard tissue has an obvious importance. For adhesive restorations to enjoy any level of success there is a prerequisite for the availability of a copious quantity and quality of tooth enamel. While improvements in adhesive technology have culminated in bonding agents which may offer superior bond strengths of resin to dentine than in past years, the bond strength is by no means comparable to that which can be achieved to enamel.

In cases where there is little remaining coronal tissue, thought may need to be given to clinical crown lengthening or the use or undertaking elective root canal therapy of affected teeth followed by the provision of post retained restorations. The drawbacks of surgical crown lengthening will be discussed in more detail in paper 3.

The use of metal posts has been well documented to be associated with the risks of root fracture, which may be exaggerated in cases where due to parafunctional habits, excessive occlusal loading has been applied. The more recent advent of fibreresin posts has been suggested to overcome this concern. However, a study by Mehta and Millar ${ }^{17}$ showed that the use of such resin-fibre posts in areas of high lateral loading, such as on anterior teeth, particularly in partially dentate patients or those displaying habits of bruxism, is associated with a high risk of fracture of the post, commonly at the post-core interface.

In extreme cases of anterior maxillary the only remaining options may be to consider the use of an overdenture or overlay denture, conventional denture or indeed dental implants.

The aesthetic requirements of the patient are also important to consider. The use of metal palatal veneers may result in a dulled appearance of the restored tooth (even with the advent of opaque/tooth coloured resin lutes) or even the visible display of metal on incisal edges. It is often very difficult to consistently gain superior aesthetic outcomes by the use of resin composite, even with the use of layering techniques; such materials may discolour, wear and chip away.
The application of conventional porcelain fused to metal restorations may result in visible 'black margins'. When used in conjunction with surgical crown lengthening, un-aesthetic 'black triangular spaces' often result, which may be undesirable.

In summary, there are arrays of factors to consider when planning the restoration of a worn anterior maxillary dentition. Comprehensive treatment planning and patient consent are vital factors to success.

\section{LOCALISED ANTERIOR MANDIBULAR TOOTH WEAR}

According to Milosevic, ${ }^{18}$ where only lower anterior teeth are affected by the process of pathological tooth wear, then the processes of prevention and monitoring will suffice. If, however, both upper and lower teeth are affected, then space should be gained through the process of the Dahl concept and the lower dentition restored before the upper. Localised anterior mandibular wear is often seen among patients who have been provided with maxillary metallo-ceramic crowns, particularly with porcelain occluding surfaces which have been adjusted to accommodate the occlusion and left unpolished.

The principle for restoring a localised worn lower dentition follows the same basic tenets as the restoration of worn maxillary anterior teeth. Where adequate space may be present in $\mathrm{CO}$, restorations can be placed with a conformative approach. In some cases space may be made available in centric relation (CR) to accommodate restorative materials, which would avoid increasing the vertical component of the patient's occlusion.

Alternatively, space can be created through the use of the Dahl concept. A fixed or removable Dahl prosthesis may be applied to the maxillary dentition to create space, or restoration fabricated resin composite can be applied directly to the affected lower anterior teeth, as illustrated by the case examples below. The latter may be retained as definitive restorations, as the treatment of choice for the medium term. Given the diminutive nature of these teeth, the preparation of the latter to receive full coverage conventional extra-coronal restorations is likely to have a long term deleterious effect on the prognosis of these teeth. ${ }^{10}$

A very successful short term prognosis has been described for the use of direct 

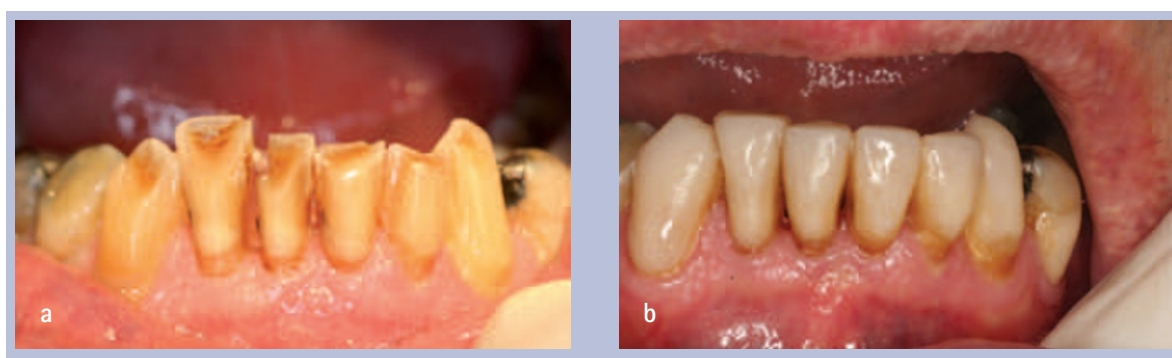

Figs 7a-b Clinical case 6 . Shows an example of lower anterior tooth wear treated using resin composite, applied 'free hand'
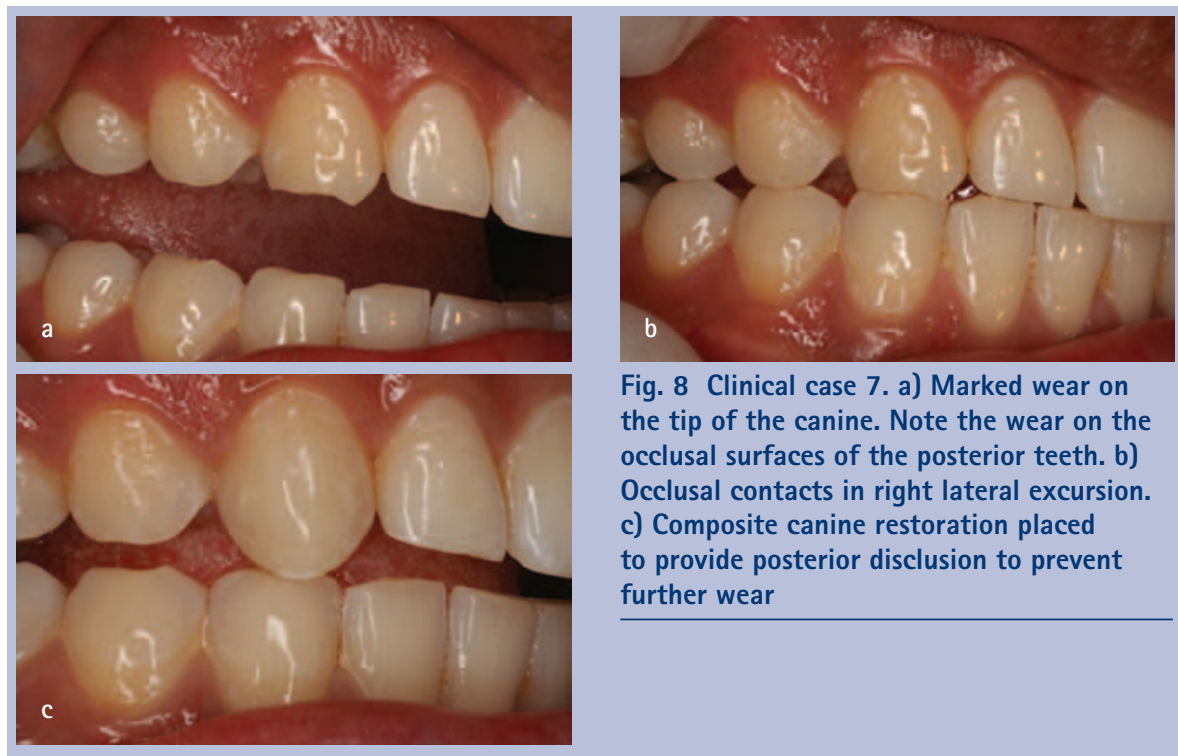

Fig. 8 Clinical case 7. a) Marked wear on the tip of the canine. Note the wear on the occlusal surfaces of the posterior teeth. $b$ ) Occlusal contacts in right lateral excursion. c) Composite canine restoration placed to provide posterior disclusion to prevent further wear

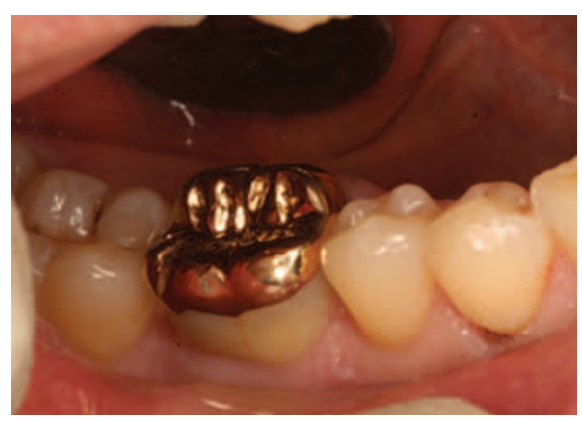

Fig. 9 Clinical case 8: localised posterior tooth wear, treated by the application of an adhesive Type III cast gold onlay

composite resin when placed in supraocclusion, utilising the Dahl approach to restore the presence of a worn lower anterior dentition. ${ }^{11}$ In the latter study the vertical occlusal dimensions of the cases included were increased in the range of 0.5 to $5.0 \mathrm{~mm}$.

Clinical case 6, Figure 7, is an example of a case treated by the means of direct resin composite applied using a 'free hand technique'. The success of latter technique is considerably dependant on operator skill. An alternative, novel approach for restoring worn lower anterior teeth will be discussed further in paper 4 .
The application of less invasive, dentine bonded crowns may have a promising role in the management of worn lower anterior teeth. Dentine bonded crowns may be defined as 'an all ceramic crown bonded to dentine (and any available enamel) using a resin based luting material with the bond being mediated by the use of an adhesive bonding system and a micro-mechanically retentive ceramic surface. ${ }^{19}$ Effectively, the latter take the form of a complete porcelain veneer, whereby strength is derived by bonding to the underlying tissue.

Burke $^{20}$ has published a case report describing the successful use of dentine bonded crowns fabricated from feldspathic porcelain (treated by hydrofluoric acid) to treat a case of severe tooth wear in a bulimic patient. Minimal tooth reduction was undertaken, involving an occlusal clearance of $1.0 \mathrm{~mm}$; preparations were finished with a knife-edged margin placed at gingival level. Dentine bonded crowns offer the merits of superior aesthetics (as they do not have a metal substructure or opaque porcelain sub-layer), furthermore the ability to bond to teeth has a considerable advantage in not only providing a patent marginal seal but where there has been copious loss of hard tooth tissue, particularly where the residual tooth structure has an over-tapered presentation. The latter forms of crown have also been reported to provide a satisfactory level of fracture resistance. ${ }^{21}$ However, the risks of fracture among patients who display parafunctional tooth grinding habits must remain a concern. ${ }^{20}$ However, their application is costly, time consuming and such restorations are unsuitable where preparations extend subgingivally. It has been suggested that the presence of a 'gingival enamel ring' is key to minimising their relative strength(s). ${ }^{7}$

Dentine bonded crowns may, however, have an interesting application in the management of wear cases, which may have previously been stabilised by the application of direct resin composite, yet there is a demand by the patient for a lower maintenance, relatively conservative, more aesthetic alternative.

Finally, as with anterior maxillary teeth, conventional crowns can be used to restore the above teeth in conjunction with procedures such as surgical crown lengthening.

\section{LOCALISED POSTERIOR WEAR}

It has been suggested that for cases of localised wear, affected teeth should be monitored and accepted, with the aim of restorative care being to provide posterior disclusion and canine guidance. ${ }^{18}$ For canine guided occlusions, resin composite can be added superiorly to the centric stop either directly (perhaps with the aid of a diagnostic waxin), so as to insure posterior tooth separation on lateral excursive and protrusive mandibular movements, to prevent the affected tooth from further wear. Alternatively an indirect restoration may be applied.

Clinical case 7, Figure 8 shows an example of a canine riser, placed 'free hand' to protect the posterior dentition from further wear, by ensuring posterior tooth disclusion upon lateral and protrusive mandibular movements.

Metal adhesive onlays, which will be discussed further in paper 3, may offer considerable potential (particularly when placed in an initial supra-occlusal position) ${ }^{22}$ to treat cases of localised posterior tooth wear. Clinical case 8, Figure 9, is an example of a gold adhesive onlay, 
fabricated from Type III cast gold alloy, cemented in supra-occlusion using Panavia EX (Kuraray, Japan), to treat a case of localised posterior tooth wear. The longevity of an adhesive onlay may be improved by the addition of resin composite to the guiding tooth, to reduce the effect of occlusal loads on the restoration during lateral and protrusive mandibular movements.

The placement of restorations in supraocclusion should be avoided among periodontally involved or endodontically treated teeth, as well as among cases which display signs of limited eruptive potential or TMJ dysfunction.

The use of resin composite onlays has been shown by Bartlett et al. ${ }^{23}$ to be associated with a high level of failure, with a failure rate of $28 \%$ for direct composite onlays and $21 \%$ for indirectly fabricated composite onlays after an observation period of three years (when placed at an increased vertical dimension). While the authors concluded that resin composite (regardless of whether restorations are fabricated directly or indirectly) is not suitable for the restoration of worn posterior teeth, they pointed out that the materials used as part of their investigation were of a microfilled variety; the use of hybrid materials and the provision of a post operative occlusal splint may have helped to improve the prognostic outcome. Indeed, a recent study has shown a favourable outcome for the application of direct resin composite restorations when used to treat tooth wear also affecting the posterior occluding surfaces, where a hybrid based material has been used (over a medium term observation period). ${ }^{24}$ The application of composite resin also provides a clinical advantage of allowing the patient to accommodate the occlusal changes, as well as enabling the clinician to make any changes if required. Once the prescription is confirmed then the composite resin restoration can be readily replaced with a restorative material with better long term durability.

\section{SUMMARY}

While the majority of cases presenting with tooth wear in general practice can be very successfully managed by the prescription of appropriate preventative means, undoubtedly there will be a small proportion of such cases which will require active restorative intervention.

Where possible, reversible, additive techniques should be applied to restore worn dentitions (at least in the short to medium term). Conventionally retained indirect restorations may be applied where adhesive resin bonded restorations may be inappropriate or display recurrent failures; however, it is paramount that the patient has a clear understanding of the implications of providing them with conventional indirect restorations (in particular the levels of tooth reduction[s] required) and the potential risks to the pulp health.

Discussed in this paper are cases of localised wear. Paper 3 will include a detailed description (illustrated by case examples) of the management of generalised wear cases and an overview of the materials and restorative techniques which can be applied to treat cases of tooth wear.

1. Allen P. Use of tooth coloured restorations in the management of tooth wear. Dent Update 2003; 30: 550-556.

2. Sundaram G, Moazzez R, Bartlett D. Trial protective effect of fissure sealants in vivo on the palatal surfaces of anterior teeth, in patients suffering from erosion. J Dent 2011; 39: 26-29.

3. Chu F, Botelho M, Newsome P, Chow T, Smales R. Restorative management of the worn dentition 2 : localised anterior tooth wear. Dent Update 2002; 29: 214-222.

4. Berry D, Poole D. Attrition: possible mechanisms of compensation. J Oral Rehabil 1976; 30: 201-206.

5. Saunders W, Saunders E. Prevalence of peri-radicular periodontitis associated with crowned teeth in an adult Scottish subpopulation. BrDent J 1998: 185: 137-140.

6. Edlehoff D, Sorenssen J. Tooth structure removal associated with various preparation designs for anterior teeth. J Prosthet Dent 2002; 87: 503-509.

7. Poyser N, Porter R, Briggs P, Kelleher M. Demolition experts: management of the parafunctional patient: 2. Restorative management strategies. Dent Update 2007; 34: 262-268.

8. Levin E. Dental aesthetics and the golden proportion. J Prosthet Dent 1978; 40: 244-252.

9. Dahl B, Krungstad O, Karlsen K, An alternative treatment of cases with advanced localised attrition. J Oral Rehabil 1975; 2: 209-214.

10. Dahl B, Krungstad O. Long term observations of an increased occlusal face height obtained by a combined orthodontic/prosthetic approach. J Oral Rehabil 1985: 12: 173-170.

11. Poyser N, Porter R, Briggs P, Chana H, Kelleher M. The Dahl concept: past, present and future. Br Dent J 2005; 198: 669-676.

12. Hemmings K, Darbar U, Vaughn S. Tooth wear treated with direct composite at an increased vertical dimension: results at 30 months. J Prosthet Dent 2000; 83: 287-293.

13. Ibbetson $R$, Setchell $D$. Treatment of the worn dentition; 2. Dent Update 1989; 16: 300-307.

14. Ricketts R. Bioprogressive therapy as an answer to orthodontic needs. Part II. Am J Orthod 1976; 70: 359-397.

15. Welbury R. A clinical study of microfilled composite resin for labial veneers. Int J Paediatr Dent 1991; 1:9-15.

16. Redman C, Hemmings K, Good J. The survival and clinical performance of resin based composite restorations to treat localised anterior tooth wear. Br Dent J 2003; 194: 566-572.

17. Mehta S B, Millar B J. A comparison of the survival of fibre posts cemented with two different resin systems. Br Dent J 2008; 13: 1-7.

18. Milosevic A. Tooth wear: management. Dent Update 1998; 25: 50-55.

19. Resinkior $\mathrm{H}$. The dentine bonded porcelain crown In Clinical procedures manual. Cedar Rapids: Dental Prosthetic Services Inc., 1987.

20. Burke F. Treatment of loss of tooth substance using dentine bonded crowns: report of a case. Dent Update 1998; 25: 235-240.

21. Burke F, Qualtrough $A$, Hale R. The dentine bonded ceramic crown: an ideal restoration. Br Dent J 1995 179: 115-121.

22. Chana H, Kelleher M, Briggs $P$, Hooper R. Clinical evaluation of resin bonded gold alloy veneers. J Prosthet Dent 2000; 83: 294-300.

23. Bartlett D, Sundaram G. An up to 3-year randomized clinical study comparing indirect and direct resin composite used to restore worn posterior teeth. Int J Prosthodont 2006; 19: 613-617.

24. Schmidlin P, Filli T, Imfeld C, Tepper S, Attin T. Three-year evaluation of posterior vertical bite reconstruction using direct resin composite a case series. Oper Dent 2009; 34: 102-108. 